\title{
An optically pumped, highly polarized cesium beam for the study of spin-dependent electron scattering
}

\author{
G. Baum, B. Granitza *, S. Hesse, B. Leuer, W. Raith, K. Rott, M. Tondera, and B. Witthuhn \\ Fakultät für Physik, Universität Bielefeld, W-4800 Bielefeld 1, Federal Republic of Germany
}

Received 1 July 1991

\begin{abstract}
We have set up an atomic beam of cesium for the study of spin-dependent electron-cesium scattering. The beam is produced by an effusive oven with continuous recirculation of the condensed metal. The beam is optically pumped by circularly polarized light from two laser diodes tuned to the $6^{2} S_{1 / 2}(F=3)$ $\rightarrow 6^{2} P_{3 / 2}\left(F^{\prime}=4\right)$ and $6^{2} S_{1 / 2}(F=4) \rightarrow 6^{2} P_{3 / 2}\left(F^{\prime}=5\right)$ transitions, respectively. Nearly all atoms are transferred into the $F=4, m_{F}=+4$ or $m_{F}=-4$ Zeeman level of the ground state, depending on the sense of circular polarization of the pumping light. The population distribution in the optically pumped beam is analyzed in terms of the $m_{J}=-1 / 2$ and $m_{J}=+1 / 2$ components with a SternGerlach magnet. We find the atomic polarization to be very close to unity at a density of $8 \times 10^{8}$ atoms $/ \mathrm{cm}^{3}$ in the scattering center. The polarization decreases slightly with increasing density of the cesium beam due to radiation trapping. A spin flipper serves as a means of polarization reversal, introducing no systematic errors in the spin asymmetry measurements. Lock-in technique is used to stabilize the atomic beam polarization by detecting fluorescence light signals.
\end{abstract}

PACS: $34.80 . \mathrm{Nz} ; 35.80 .+\mathrm{s}$

\section{Introduction}

Our research interest is focused on the investigation of spin effects in the elastic and inelastic scattering of electrons from atoms. Atomic physics experiments, utilizing polarized electrons and polarized atomic beams, have been carried out in several laboratories, covering electron-impact ionization of $\mathrm{H}, \mathrm{He}\left(2^{3} S\right), \mathrm{Li}, \mathrm{Na}, \mathrm{K}$, electron-impact excitation of $\mathrm{Li}, \mathrm{Na}$, and elastic scattering on H, Li (cf. reviews of Raith 1988, Baum et al. 1990, Kessler 1991). For these light atoms spin-dependent ef-

* Present address: School of Physical Sciences, The Flinders University of South Australia, Bedford Park, SA 5042, Australia fects result from electron exchange. In the scattering from heavy atoms, however, relativistic effects such as the spin-orbit interaction play an important role. For unpolarized heavy atoms this leads to the well known Mott scattering. If both exchange and spin-orbit interactions are present, as in the scattering of polarized electrons from polarized heavy atoms, interference between these two interactions can be expected (Farago 1974, Walker 1974, Ob'edkov and Van der Wiel 1978, Bartschat 1990).

For studies of such interferences a highly polarized beam of cesium atoms is desirable, since cesium is the heaviest alkali metal. Unfortunately the classical method of polarizing atomic beams, i.e., state selection in strong inhomogeneous magnetic fields, is not applicable here because of the large number of Zeeman levels of the atom, which result from the high nuclear spin of $I=7 / 2$, and because of the strong hyperfine interaction. Both facts would lead to a low atomic polarization when following that method. Moreover it would be very hard to determine its value precisely (see e.g. Baum et al. 1972).

We therefore chose laser optical pumping as the most suitable method to achieve a high degree of atomic polarization. For this it is necessary to pump both hyperfine levels of the ground state which are about $9.2 \mathrm{GHz}$ apart in frequency. If only one laser is available acusto-optical modulators can be used to produce two light beams with the resonant frequencies. This technique has been applied to polarize a lithium atomic beam (Baum et al. 1980) but for technical reasons it is only suitable for hyperfine structure splittings of up to $2 \mathrm{GHz}$. Another possibility for optical pumping with only one laser beam is frequency modulation. This has been done for cesium by Watts and Wiemann (1986) who exploited the excellent frequency characteristics of laser diodes. We decided to use two laser diodes to pump both hfs levels simultaneously as this appeared to us to be technically simpler.

Because of the large number of Zeeman states of cesium a precise determination of the achieved atomic polarization is rather difficult. However, this problem diminishes if one is able to reach a polarization very close to unity. Optical methods have been used, measuring 
the difference in the absorption of right and left circularly polarized light in the transition $6^{2} S_{1 / 2}(F=4)$ $\rightarrow 6^{2} P_{3 / 2}\left(F^{\prime}=5\right)$ (Watts and Wiemann 1986). We use a Stern-Gerlach magnet which has the advantage of being a very stable and reliable instrument. From the measured beam profiles, we deduce an upper and a lower limit for the degree of polarization by means of two assumptions about extreme population distributions in the unresolved hfs components.

\section{Cesium oven}

The high atomic mass of cesium requires a relatively high throughput in the oven to achieve a certain density in the beam. Because of the high cost of the cesium metal and the dangers in handling large quantities of it, an oven is needed that recycles and re-uses as much of the emitted cesium metal as possible. This will provide long operating times. A further advantage of such a device is the fact that the vacuum chamber and its inner parts are seldom exposed to air and, therefore, cleaning chores are reduced accordingly. Figure 1 shows the design of our oven system.

Cesium vapor ascends from the oven body through a pipe to a $1-\mathrm{mm}$ diameter orifice. A collimator with a diameter of $2 \mathrm{~mm}$ forms the beam. The temperature of the recycling funnel, including the front face of the collimator, is controlled to lie just above the melting point of cesium $\left(28^{\circ} \mathrm{C}\right)$. The cesium which does not pass

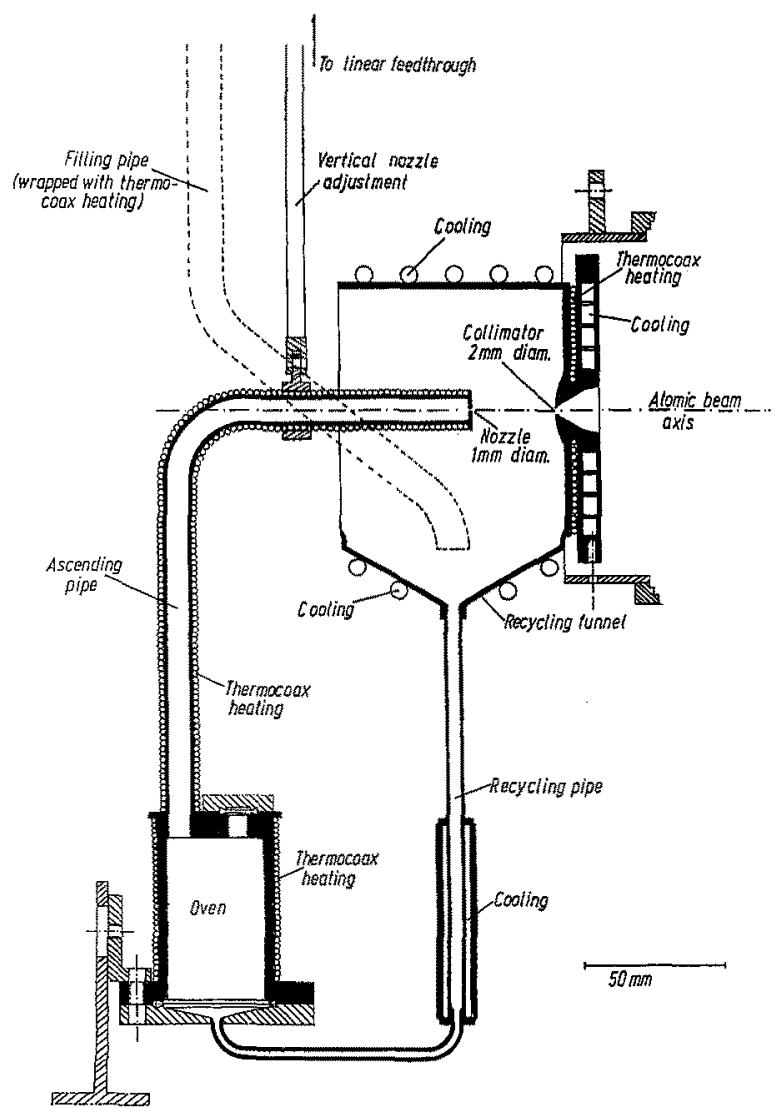

Fig. 1. Cross section of the recirculating Cs oven the collimating aperture condenses on these surfaces, stays liquid, and flows down into the recycling pipe and from there back into the oven. Since the bottom part of the recirculating pipe is filled with cesium, cooling provisions are also necessary in this part of the system in order to prevent cesium from evaporating back up the pipe. Cooling is achieved by circulating water from a bath thermostat through pipes attached to the various devices.

The nozzle, the ascending pipe and the oven itself can be heated separately, allowing temperature settings which will prevent blockage and which will reduce the dimer content of the atomic beam. For the latter the nozzle is overheated by about $80^{\circ} \mathrm{C}$ with respect to the oven. The collimator can be heated to clean its aperture in case of clogging, but this has not happened so far. As encased resistive heating wire we use Thermocoax (Philips Corp.) of $2 \mathrm{~mm}$ diameter.

The oven assembly is mounted inside a vacuum chamber, which is sealed with Viton gaskets and pumped by a $500 \mathrm{l} / \mathrm{s}$ turbomolecular pump. The oven chamber can be isolated from the rest of the apparatus by a gate valve in the atomic beam line. While the collimator is fixed with respect to a chamber flange, the nozzle can be moved in a plane perpendicular to the beam direction by means of rods attached to linear feedthroughs. This allows compensation for misalignment caused by thermal expansion.

We use a separately pumped vacuum lock to fill cesium into the oven. After cracking the ampoule under vacuum (with valve V1 open and valve V2 closed) the cesium is evaporated from the bellows assembly (see Fig. 2) through the filling pipe by heating these parts to about $350^{\circ} \mathrm{C}$. It finally condenses on the cold surfaces of the recycling funnel. Once the lock is emptied of cesium, V1 is closed and the bellows detached for cleaning. If the cesium in the oven has been used up, the refilling can be carried out by the procedure described above.

We are able to achieve a stable atomic beam with a density of $8 \times 10^{8}$ atoms $/ \mathrm{cm}^{3}$ at the location of the scattering region which is a distance of about $1.6 \mathrm{~m}$ away from the nozzle. The intensity profile was measured by

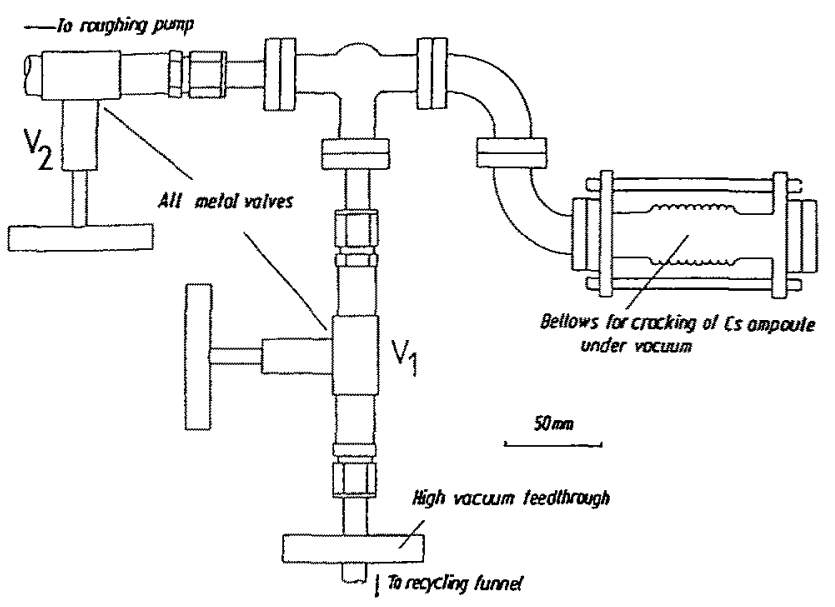

Fig. 2. Assembly for loading the oven 
means of a Langmuir-Taylor detector. With a filling of $5 \mathrm{~g}$ of cesium we can continuously operate for about $200 \mathrm{~h}$. Densities in excess of $10^{9}$ atoms $/ \mathrm{cm}^{3}$ can easily be achieved at the expense of operating time.

\section{Optical pumping}

We use optical pumping on the cesium- $\mathrm{D}_{2}$ line $(852.1 \mathrm{~nm})$ since laser diodes suitable for this wavelength are easily available. Figure 3 shows the energy level diagram for this transition. One of the laser diodes is tuned to the $6^{2} S_{1 / 2}(F=3) \rightarrow 6^{2} P_{3 / 2}\left(F^{\prime}=4\right)$ transition. The selection rules allow a decay back into both hfs levels of the ground state. Eventually all atoms from the $F=3$ levels of the ground state will be transferred by the circularly polarized light to the $F=4$ part of the ground state. The second laser diode pumps the $6^{2} S_{1 / 2}(F=4)$ $\rightarrow 6^{2} P_{3 / 2}\left(F^{\prime}=5\right)$ transition with circularly polarized light. For the present discussion we assume pumping with $\sigma^{+}$light, allowing only excitations with $\Delta m_{F}=+1$ : After one pumping cycle of excitation and subsequent decay an atom will be in the $F=4$ ground state with its magnetic quantum number either unchanged or increased by 1 or 2 . Several pumping cycles (their number depending on the original Zeeman state) will bring the atoms in the wanted state $F=4, m_{F}=+4$ which carries full polarization.

Hitachi HLP1400 laser diodes are used, providing a single-mode output power of $10 \mathrm{~mW}$ with a band width of $30 \mathrm{MHz}$ (FWHM). Tuning to the appropriate wavelength is done by adjusting the case temperature of the semiconductor diode. A tuning range of $\pm 3 \mathrm{~nm}$ with respect to the wavelength specified at room temperature is possible. Fine tuning can then be achieved by changing the injection current. This is usually done in order to tune the diodes to the required hyperfine transition.

Figure 4 shows schematically the set-up for the optical pumping. The light of the lasers is collimated to form
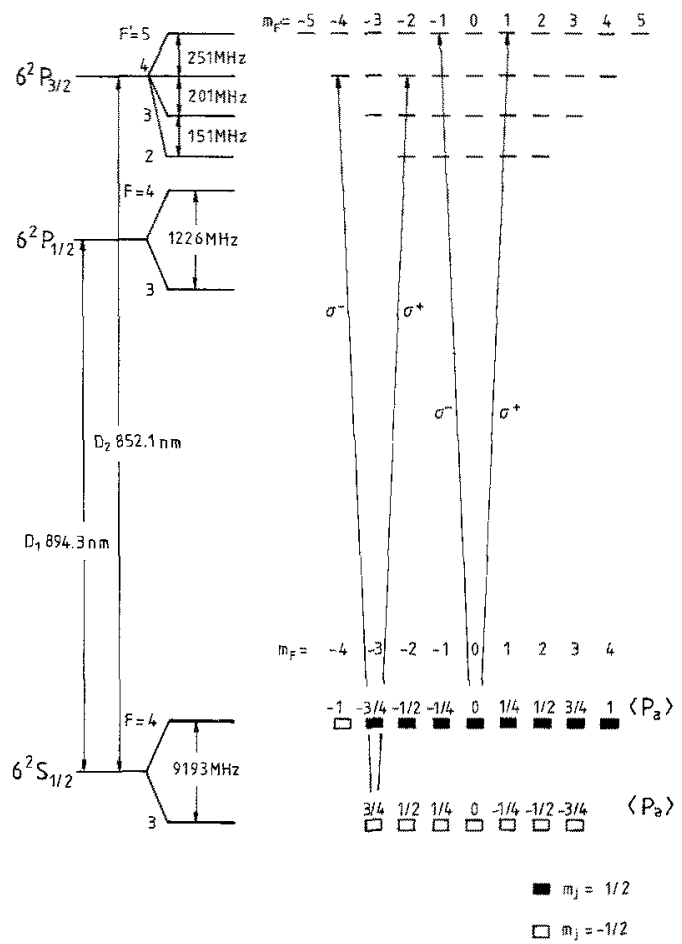

Fig. 3. Cs level diagram for explaining the optical pumping. Note that high-field state selection separates all the $F=3$ Zeeman states together with the $F=4, m_{F}=-4$ state from all the other $F=4$ states

parallel beams by using anti-reflex coated lenses of short focal length. Both beams are diverted by mirrors and interesect the atomic beam at right angles. The cross sections of the beams at the intersection is about $10 \mathrm{~mm}$, determining the length of the optical pumping region. A Glan-Thompson polarizing prism (extinction ratio $<10^{-6}$ ) serves as a linear polarizer; a quarter-wave plate (zero-order quartz) produces circularly polarized light.

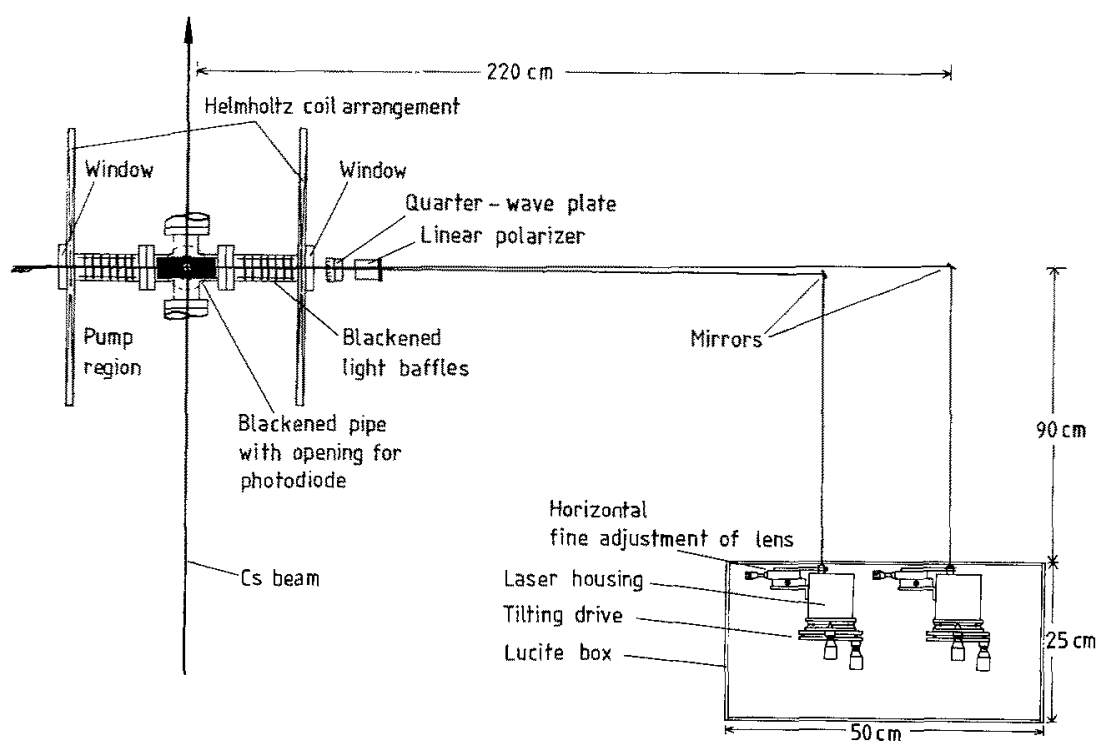

Fig. 4. Experimental arrangement for the dualwavelength optical pumping 
These elements are common to both beams. The light is circularly polarized with respect to a magnetic quantization field of $10^{-4} \mathrm{~T}$, collinear with the direction of the laser beam, produced by a Helmholtz-coil arrangement. The band width of the lasers assures that, in spite of the slight Zeeman splitting induced by the quantization field, all Zeeman states are equally well pumped. The reversal of the circular polarization of the pumping light is accomplished by simply turning the quarter-wave plate by $90^{\circ}$. A photodiode is mounted above the pumping region in order to observe the fluorescent light and identify the required hyperfine transitions.

Each laser diode is temperature stabilized utilizing a temperature controller that has been designed by following the work of Handschy (1983). Since laser diodes are very susceptible to short spikes in their injection current, which would destroy or irreversibly degrade them, a fully battery powered current supply has been developed. It stabilizes the injection current to within $1 \mu \mathrm{A}$.

The optically pumped transition $F=4 \rightarrow F^{\prime}=5$ should be a cycling transition since, according to the selection rules, the excited atoms can only decay back into the $F=4$ ground state. However, due to the relatively large bandwidth of the laser light the intensity at the frequency of the transition $F=4 \rightarrow F^{\prime}=4$ is not zero and therefore this transition will also be pumped weakly. Atoms affected such can decay into the $F=3$ ground state. This leakage has been investigated both theoretically and experimentally by McClelland and Kelley (1985) for an atomic sodium beam pumped by a dye laser. Since these authors pumped only one hyperfine level considerable leaking was found especially at high laser power. In our experiment the $F=3$ ground state is pumped simultaneously with the $F=4$ ground state. Therefore an atom "lost" by leaking will be pumped back into the $F=4$ level.

Two lock-in amplifiers stabilize the frequencies for the diodes. This is accomplished by a sinusoidal modulation of the injection currents for the laser diodes leading to a weak modulation of the corresponding laser frequencies. The modulation has negligible influence on the resulting polarization. The laser for the transition $6^{2} S_{1 / 2}(F=3) \rightarrow 6^{2} P_{3 / 2}\left(F^{\prime}=4\right)$ is modulated at about $700 \mathrm{~Hz}$, the laser for the transition $6^{2} S_{1 / 2}(F=4)$ $\rightarrow 6^{2} P_{3 / 2}\left(F^{\prime}=5\right)$, at about $1900 \mathrm{~Hz}$. The oberserved fluorescence light originates almost exclusively from the transition $F^{\prime}=5 \rightarrow F=4$, nevertheless both modulation frequencies are contained in it. This occurs because the population of the $F=4$ ground state varies periodically by the selective excitation of the $F=3$ ground state. The fluorescence signal is fed into the two lock-in amplifiers, locked at 700 and $1900 \mathrm{~Hz}$, respectively. The lock-in amplifiers average the fluorescence signal in the following way: The intensity during the negative half-wave of the frequency-modulating sine wave is substracted from that during the positive half-wave. Thus if the laser matches the resonance frequency at the zero transition of the modulating sine wave, the amplifier signal is zero; otherwise, a positive or negative signal results which is proportional to the frequency deviation and serves to adjust the current for the laser accordingly. This technique al- lows operations with stable polarization of the atomic beam for several hours.

A longitudinal guiding magnetic field of about $10^{-5} \mathrm{~T}$ is applied to the atomic beam in the region behind the optical pumping zone. This is necessary in order to prevent the atoms from undergoing depolarizing Majorana transitions between adjacent Zeeman states.

\section{Polarization analysis}

From the optically pumped cesium beam a small portion is selected by a $0.3 \mathrm{~mm} \times 4 \mathrm{~mm}$ vertical slit and then passed through a Stern-Gerlach magnet. The magnetic field between the $100 \mathrm{~mm}$-long pole pieces is everywhere greater than $0.5 \mathrm{~T}$. Therefore the hyperfine coupling scheme is no longer valid and the Zeeman states are not degenerate. The atoms are deflected perpendicular to the initial beam direction. More specifically, atoms with magnetic quantum number $m_{J}=+1 / 2$ (i.e., $F=4$, $\left.m_{F}=-3, \ldots,+4\right)$ are deflected to the right while those with the magnetic quantum number $m_{J}=-1 / 2$ (i.e., $F$ $=3, m_{F}=-3, \ldots,+3$ and $F=4, m_{F}=-4$ ) are deflected to the left, when looking in the downstream direction. By using a movable Langmuir-Taylor detector the splitted beam profile beyond the Stern-Gerlach magnet can be scanned in order to get information on the population distribution achieved by the optical pumping process.

The actual degree of polarization can be evaluated on the basis of a few very well founded assumptions. Firstly we assume that both senses of circular polarization of the pumping light yield the same degree of atomic polarization. To prove the validity of this assumption a spin flipper mounted between the optical pumping region and the Stern-Gerlach magnet is used. This device, a slightly modified version of the one described by Schröder and Baum (1983), is characterized by two modes of operation. In the diabatic ("flip") mode atoms diabatically pass a zero crossing of a longitudinal magnetic guiding field and are transferred from the state $F, m_{F}$ to the state $F,-m_{F}$ which has opposite polarization (see Fig. 3). In the adiabatic ("no flip") mode a transverse magnetic field is added at the zero-crossing point, allowing the atoms to adiabatically follow the change of direction of the guiding field. In this case the polarization direction with respect to the guiding field does not change.

If optical pumping with both $\sigma^{+}$and $\sigma^{-}$light is equally effective (i.e., the above assumption is correct), $\sigma^{-}$ pumping with subsequent spin flip and $\sigma^{+}$pumping without spin flip should yield identical Stern-Gerlach profiles of the Cs-beam. Also the profiles for " $\sigma^{+}$, flip" and " $\sigma^{-}$, no flip" have to be identical. As can be seen from Fig. 5, these conditions are satisfied. Figure 5 also shows (case " $\sigma^{+}$, no flip") that in the ground state the levels with $F=3, m_{F}=-3$ to +3 and $F=4, m_{F}=-4$ have been completely emptied. On the basis of this observation one knows that, when pumping with $\sigma^{-}$light, the polarized beam contains only atoms in the levels $F=4, m_{F}=-4\left(m_{J}=-1 / 2\right)$ and $F=4, m_{F}=-3, \ldots,+3$ $\left(m_{J}=+1 / 2\right)$. 


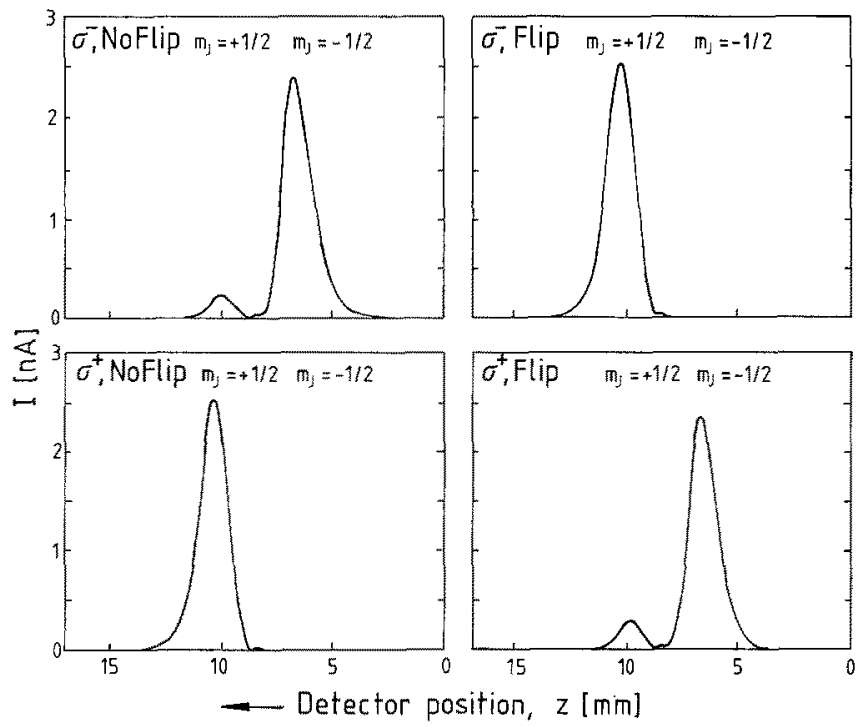

Fig. 5. Measured Stern-Gerlach beam profiles

As can be seen from the case " $\sigma^{-}$, no flip" in Fig. 5, most of the atoms are in the $m_{F}=-4$ state, which carries full polarization. The exact value of the beam polarization now depends on the distribution of a small fraction of atoms over the $F=4, m_{F}=-3, \ldots,+3$ states. At this stage two further assumptions can be made, leading to the most favorable and the most disadvantageous of possible distributions. For the best case, all atoms contributing to the $m_{J}=+1 / 2$ peak are in the state $m_{F}=-3$ with a polarization of $-3 / 4$. For the worst case all $m_{J}=+1 / 2$ atoms are evenly distributed over the sub-states $m_{F}=$ $-3, \ldots,+3$ and therefore do not contribute to the polarization. The case of a population distribution which increases with increasing $m_{F}$ value can be excluded on the basis of our rate-equation calculations. These calculations were carried out following the description of Hils et al. (1981). In conclusion, the actual distribution will lie somewhere between the two cases assumed above.

By integrating the Stern-Gerlach profile of the case " $\sigma$, no flip" and applying the above assumptions the degree of polarization, $P_{a}$, can be obtained by using the expression.

$P_{a}=\frac{\sum_{F, m_{F}}\left[N\left(\left|F, m_{F}\right\rangle\right) \cdot P_{a}\left(\left|F, m_{F}\right\rangle\right)\right]}{\sum_{F, m_{F}} N\left(\left|F, m_{F}\right\rangle\right)}$,

where $P_{a}\left(\left|F, m_{F}\right\rangle\right)$ denotes the degree of polarization of the state $\left|F, m_{F}\right\rangle$, which has the population $N\left(\left|F, m_{F}\right\rangle\right)$. For the best case assumed the modulus of the atomic beam polarization becomes

$\left|P_{a}\right|_{\max }=\frac{I\left(m_{J=-1 / 2}\right)+3 / 4 I\left(m_{J=+1 / 2}\right)}{I\left(m_{J=-1 / 2}\right)+I\left(m_{J=+1 / 2}\right)}$

and for the worst case

$\left|P_{a}\right|_{\min }=\frac{I\left(m_{J=-1 / 2}\right)}{I\left(m_{J=-1 / 2}\right)+I\left(m_{J=+1 / 2}\right)}$, where $I\left(m_{J=+1 / 2}\right)$ denotes the integration value of the respective profile.

This procedure provides an interval, from $\left|P_{a}\right|_{\min }$, to $\left|P_{a}\right|_{\max }$, in which the actual value of the atomic polarization lies. We quote the median of the interval as atomic polarization while half of its width is quoted as uncertainty.

As can be seen from figure 5 there is a small difference in polarization between the cases " $\sigma^{-}$, no flip" and " $\sigma^{+}$, flip", with the polarization in the latter case slightly smaller. This we attribute to the efficiency of the spin flipper which is about $98 \%$.

\section{Results}

We recorded Stern-Gerlach profiles of the optically pumped $\mathrm{Cs}$ beam for a wide range of beam densities and evaluated the atomic polarization by integration of the Stern-Gerlach profiles. Table 1 gives the results for various beam densities. Note that the degree of polarization decreases with increasing density, while its uncertainty increases, reaching values of up to $\pm 5 \%$ at densities of $2 \times 10^{9}$ atoms $/ \mathrm{cm}^{3}$. At very low densities the polarization does hardly deviate from unity. This indicates that the reason for incomplete atomic polarization at higher densities cannot be insufficient polarization of the pumping light, since this problem would be density independent. Further investigations showed that enhancing (by using an additional laser) as well as slightly attenuating the laser power does not change the observed profiles. Therefore lack of laser power cannot be the reason for incomplete polarization either. We also changed the length of the optical pumping region and could not observe an influence on the polarization.

A density dependent effect that will diminish the atomic polarization is radiation trapping, (e.g., Happer 1972). Spontaneously emitted resonance radiation, which is present as long as the atoms are in the optical pumping

Table 1. Data related to the atomic polarization for different atomic beam densities. Above: "no flip" mode. Below: "flip" mode

\begin{tabular}{|c|c|c|c|c|c|}
\hline $\begin{array}{l}\text { No } \\
\text { Flip }\end{array}$ & $\begin{array}{l}\text { Density } \\
{\left[\text { At. } \mathrm{cm}^{-3}\right]}\end{array}$ & $\begin{array}{l}\left|P_{a}\right|_{\max } \\
{[\%]}\end{array}$ & $\begin{array}{l}\left|P_{a}\right|_{\min } \\
{[\%]}\end{array}$ & $\begin{array}{l}\left|P_{a}\right| \\
{[\%]}\end{array}$ & $\begin{array}{l}A\left|P_{a}\right| \\
{[\%]}\end{array}$ \\
\hline a) & $1.9 \cdot 10^{9}$ & 96.2 & 85.4 & 90.8 & \pm 5.4 \\
\hline b) & $8.6 \cdot 10^{8}$ & 98.2 & 92.8 & 95.5 & \pm 2.7 \\
\hline c) & $4.2 \cdot 10^{8}$ & 98.5 & 94.3 & 96.4 & \pm 2.1 \\
\hline d) & $1.0 \cdot 10^{8}$ & 99.2 & 96.8 & 98.0 & \pm 1.2 \\
\hline e) & $1.9 \cdot 10^{7}$ & 99.8 & 99.1 & 99.45 & \pm 0.35 \\
\hline Flip & $\begin{array}{l}\text { Density } \\
{\left[\text { At. } \mathrm{cm}^{-3}\right]}\end{array}$ & $\begin{array}{l}\left|P_{a}\right|_{\max } \\
{[\%]}\end{array}$ & $\begin{array}{l}\mid P_{a l m i n} \\
{[\%]}\end{array}$ & $\begin{array}{l}\left|P_{a}\right| \\
{[\%]}\end{array}$ & $\begin{array}{l}\Delta\left|P_{d}\right| \\
{[\%]}\end{array}$ \\
\hline a) & $1.9 \cdot 10^{9}$ & 95.2 & 82.6 & 88.9 & \pm 6.3 \\
\hline b) & $8.6 \cdot 10^{8}$ & 97.8 & 91.4 & 94.6 & \pm 3.2 \\
\hline c) & $4.2 \cdot 10^{8}$ & 97.4 & 90.8 & 94.1 & \pm 3.3 \\
\hline d) & $1.0 \cdot 10^{8}$ & 97.2 & 90.6 & 98.9 & \pm 3.3 \\
\hline e) & $1.9 \cdot 10^{7}$ & 99.0 & 96.2 & 97.6 & \pm 1.4 \\
\hline
\end{tabular}


region (and even in a limited region behind it), can induce transitions with a $\Delta m_{F}$ different from the one induced by the circularly polarized pumping light. If a fully polarized atom absorbs one of these more or less arbitrarily polarized photons it can be depolarized. The intensity of fluorescence light depends on the density of the atomic beam in the optical pumping region and, therefore, the probability of depolarization is dependent on the beam density. The effect might even be more important in the region where the atoms have just left the pumping region, i.e., where the (correctly polarized) pumping light is no longer present, but the diffuse fluorescent radiation still is. We checked the influence of radiation trapping by setting up an additional optical pumping region, approximately $100 \mathrm{~mm}$ behind the main one, provided with light from an additional laser tuned to the $F=4 \rightarrow F^{\prime}=5$ transition. If the power of this laser is $10 \mathrm{~mW}$ as in the main pumping region the polarization values do not change. However, if the intensity of the additional laser is attenuated to about $2 \mathrm{~mW}$ the atomic polarization increases considerably. This effect is due to the additional pumping done here to those atoms which are not yet completely polarized, but without creating as much fluorescence background radiation as in the main pumping region. However, if the light in the main pumping region were attenuated to a power level of $2 \mathrm{~mW}$, this would not suffice to completely pump the atomic beam.

\section{Conclusions}

Our cesium beam with its high polarization value and its stable intensity is well suited to study the spin dependence of polarized electron collisions on this heavy atom. The recirculating oven provides reasonable beam density combined with low cesium consumption and with high reliability. Laser diodes have shown to be easy to use. They are reliable devices for the purpose of optical pumping, yielding a high degree of atomic polarization. The analysis of the atomic polarization by means of a Stern-Gerlach magnet provides an accurate value in a relatively simple way. It also allows a continuous monitoring of the polarization. Measurements of the spin asymmetry in the total ionization cross section have been made (Baum et al. 1991).

This work has been supported by the Deutsche Forschungsgemeinschaft (SFB 216) and the University of Bielefeld.

\section{References}

1. Bartschat, K.: J. Phys. B 23, 2341 S (1990)

2. Baum, G., Lubell, M.S., Raith, W.: Phys. Rev. A5, 1073 (1972)

3. Baum, G., Caldwell, C.D., Schröder, W.: Appl. Phys. 21, 121 (1980)

4. Baum, G. et al.: Abst. ICPEAXVII, p. 106, Brisbane 1991

5. Baum, G., Freienstein, P., Frost, L., Granitza, B., Raith, W., Steidl, H.: In: Proc. Int. Symp. Correlation and Polarization in Electronic and Atomic Collisions, Neill, P.A., Becker, K.H., Kelley, M.H. (eds.), NIST Special Publication 789, p. 121, Gaithersburg, USA 1990

6. Farago, P.S.: J. Phys. B7, L 28 (1974)

7. Handschy, M.A.: J. Phys. E13, 998 (1983)

8. Happer, W.: Rev. Mod. Phys, 44, 169 (1972)

9. Hils, D., Jitschin, W., Kleinpoppen, H.: Appl. Phys. 25, 39 (1981)

10. Kessler, J.: Adv. At. Mol. Opt. Phys. 27, 81 (1991)

11. McClelland, J.J., Kelley, M.H.: Phys. Rev. A 31, 3704 (1985)

12. Ob'edkov, V.D., Wiel, M.J. Van der: J. Phys. B11, L329 (1978)

13. Raith, W.: In: Fundamental processes of atomic dynamics, p. 429, Briggs, J., Kleinpoppen, H., Lutz, H. (eds.). New York: Plenum Press 1988

14. Schröder, W., Baum, G.: J. Phys. E16, 52 (1983)

15. Walker, D.W.: J. Phys. B7, L489 (1974)

16. Watts, R.N., Wiemann, C.E.: Opt. Comm. 57, 45 (1986) 